\title{
$\checkmark$ Pregnancy and multiple sclerosis
}

PATIENT'S

CHOICE

${ }^{1}$ Department of Neurology,

Norfolk \& Norwich University

Hospital, Norwich, UK;

${ }^{2}$ Institute for Women's Health,

University College London

Hospitals, London, UK

Correspondence to:

Dr M Lee, Department of

Neurology, Norfolk \& Norwich

University Hospital, Norwich,

UK; martin.lee@nnuh.nhs.uk

Received 6 December 2007

Revised 1 July 2008

Accepted 27 July 2008

\section{Lee, ${ }^{1}$ P O'Brien²}

\section{ABSTRACT}

At any one time, there are around 20000 women of childbearing age with multiple sclerosis (MS) in the UK who may be considering having children. Neurologists can be asked for information from both patients and obstetric colleagues on a range of topics related to pregnancy and MS that extend beyond the well-known implications for relapse risk. This article aims to provide a brief overview for the general neurologist of the most commonly encountered issues and questions including those occasionally related to pregnancy management. The takehome message is that pregnancy does not hold adverse risks for the majority of patients with MS, or vice versa.

Multiple sclerosis (MS) is a chronic inflammatory disease of the central nervous system with onset typically in the second to third decade and is twice as prevalent in females as males. In the past, there has been speculation that pregnancy, together with other stressful life events, adversely affects the risk of relapse $e^{1}$ or the course of the disease. ${ }^{2}$ In fact, pregnancy appears to be associated with a temporary beneficial immune state for patients with MS partly mediated through an effect on T lymphocyte subsets. This effect may have relevance for autoimmune diseases in general.

The pathogenesis of MS remains incompletely understood but involves a maladaptive humoral and cell-mediated immune response to an as-yet undetermined antigen(s). The popular model begins with peripheral $\mathrm{T}$ cell sensitisation in response to macrophage presentation of a foreign "myelin mimicking" antigen in association with MHC class II. ${ }^{3}$ This results in peripherally activated $T$ cells expressing, and recognising, vascular adhesion molecules facilitating their entry to the central nervous system (CNS). Inside the CNS, activated $\mathrm{T}$ cells release pro-inflammatory cytokines resulting in upregulation of local CNS microglia to antigen-presenting cells with the capacity to present self-myelin and other myelinassociated proteins. This leads to an "epitope spreading" phenomenon and further secondary activation of $\mathrm{T}$ cells triggering an autoimmune inflammatory cascade. A direct and bystander inflammatory response results in CNS conduction block, demyelination and axonal damage that variably contribute to reversible and persistent neuronal dysfunction with associated neurological disability. Other models of disease pathogenesis exist such as persistence of a foreign viral antigen as the perpetuating stimulus, ${ }^{4}$ but most models place $\mathrm{T}$ cells centre stage.

$\mathrm{T}$ cells can be subdivided into cytotoxic and helper $\mathrm{T}$ cells. The latter are associated with the MHC class II linked immune response underlying MS and subdivide into type-1 (Th-1) and type-2
(Th-2) helper cells. Th-1 cells release pro-inflammatory cytokines including IL2, INF- $\gamma$ and TNF- $\alpha$, while Th- 2 cells are broadly antagonistic, secreting IL- 6 and IL-10 which suppress the Th-1 response and drive $\mathrm{B}$ cell maturation and antibody production. ${ }^{5} \mathrm{MS}$ is believed primarily to be a Th-1 driven immune state with Th-1 associated pro-inflammatory cytokines promoting blood-brain barrier breakdown, further immune cell recruitment, myelin and axonal injury. The balance between Th- 1 and Th- 2 associated immune states therefore influences disease activity and itself appears hormonally sensitive with pregnancy favouring a Th-2 type response.

Pregnancy, by necessity, involves a relative state of immunosuppression as the fetus carries paternally derived antigens, and it is likely that high levels of oestrogen associated with pregnancy contribute to this. Oestrogen is known to be associated with a Th-2 type immune response and downregulation of microglial activity, and has been shown to suppress extrinsic allergic encephalomyeltis (EAE), an animal model of MS. ${ }^{6}$ There has been particular interest in the immunosuppressive role of oestriol, an oestrogen produced by the fetalplacental unit and detected only during pregnancy. Oestriol levels appear to mirror most closely the reduction in relapse frequency seen during the third trimester of pregnancy, and there has already been a pilot study of estriol as a therapeutic agent in non-pregnant patients with MS that reported an $80 \%$ reduction in MRI disease activity over 6 months. $^{7}$ A follow-on phase II/III clinical trial is currently under way of oestriol as add-on therapy to Copaxone in female MS patients.

Unfortunately, the immunosuppressive oestrogen profile found in pregnancy does not appear to translate into a similar protective benefit in women with lower oestrogen levels seen outside pregnancy. Oestrogen levels in non-pregnant females appear associated with microglial upregulation and a less favourable Th-1 type immune response. $^{8}$

There are a large number of other factors potentially linked with an immunosuppressive Th-2 associated immune response during pregnancy, ${ }^{910}$ an effect likely to be as beneficial to patients with other autoimmune diseases ${ }^{11}$ as it is to patients with $\mathrm{MS}^{12}$ Of these factors, it is perhaps worth singling out the hormonal form of vitamin $\mathrm{D}$, calcitriol. Calcitriol levels peak in the first trimester and fall rapidly postpartum, inversely reflecting MS disease activity. Outside pregnancy, low levels of vitamin D associated with reduced sun exposure with increasing latitude have been linked with MS susceptibility. ${ }^{13}$ The perceived immunosuppressive benefit of vitamin $\mathrm{D}$ has led to small pilot studies of vitamin D supplementation 
in patients with $\mathrm{MS},{ }^{14}$ though no firm conclusions regarding efficacy can yet be made.

In summary, hormonal and cytokine changes during pregnancy appear linked with a Th-2 type immune state likely to be beneficial for patients with autoimmune inflammatory conditions such as MS. Further knowledge of the nature of this effect may provide insight into additional treatment strategies for patients with MS.

\section{PREGNANCY AND RELAPSES}

During the first half of the twentieth century, it was generally held that pregnancy adversely affected the risk of relapse and course of the disease in patients with $\mathrm{MS}^{2}$ and therapeutic abortion was occasionally advocated. Several retrospective studies $^{15}{ }^{16}$ and more latterly results of the Pregnancy in Multiple Sclerosis (PRIMS) study ${ }^{10}$ have dispelled this myth, showing that pregnancy is probably neutral overall in terms of disease activity. The prospective PRIMS study of 227 European women with MS who had a full-term delivery reported a reduction in relapse rate during pregnancy, most marked in the third trimester, with a compensatory increase in the first postpartum trimester before returning to prepregnancy levels. An approximate $70 \%$ reduction in relapse rate was seen in the third trimester (double that of currently licensed standard disease modifying therapies (DMTs)). There was a similar increase above background prepregnancy rates for the first trimester postpartum. Overall, therefore, in the year which includes pregnancy and the 3 months after birth, the total number of relapses is similar to other years.

The clinical reduction in relapse activity during pregnancy is accompanied by evidence of a reduction in MRI activity. There was a marked reduction in new or enhancing disease activity on serial brain MRI scanning in two patients with MS who became pregnant while already enrolled on an MRI study. MRI lesion activity returned to prepregnancy levels postpartum. ${ }^{17}$

While the reduction in relapse rate during pregnancy is welcome, the risk of relapse postpartum raises questions about preventive therapy options. It is not possible to identify particular individuals at high risk for purposes of targeted therapy trials, but there have been a few small retrospective case series reports of both intravenous methylprednisolone ${ }^{18}$ and intravenous immunoglobulin ${ }^{19}$ given in the postpartum period to patients with MS. These studies suggest a reduction in relapse frequency (of $30-60 \%$ ) but do not provide an evidence base on which to recommend treatment in the absence of any controlled trial data. Current practice is to consider treatment of postpartum relapse in the usual way with a short course of methylprednisolone. This is generally felt not to have significant implications for breast feeding. Intravenous immunoglobulin is generally avoided due to the potential for neonatal exposure to pooled blood product with intravenous immunoglobulin.

\section{PREGNANCY AND MS ONSET}

MS disease onset may be reduced during pregnancy. Runmarker and Anderson ${ }^{20}$ used a near-complete incidence cohort followed up for 25 years to look at onset bouts during pregnancy in 100 females with relapse onset MS. They found there were no onset bouts during pregnancy and nine bouts in the 8 months following delivery $(p=0.004)$. Risk of onset was not different between the postpartum period and other non-pregnancy periods. In addition, nulliparous patients were over-represented at $\mathrm{MS}$ onset by $50 \%$ compared with age-matched population controls. By studying disease onset, the suggestion is that MS symptoms do not modify conception behaviour and that pregnancy may therefore be associated with some protection from MS onset.

\section{PREGNANCY AND MS PROGRESSION}

Disease progression was not affected by pregnancy in the PRIMS study, although the majority of patients had relapsing remitting disease, and follow-up was relatively short. Most other studies have not found any adverse effect of pregnancy on disease progression, ${ }^{21}$ and some reports suggest a favourable effect with increased time to progressive disease onset following pregnancy. ${ }^{20}$ However, despite age and disability matching, it is difficult to remove conceptive behaviour bias from these studies. It does seem reasonable to conclude that there is at least no adverse effect of pregnancy on subsequent disease course.

\section{MS AND PREGNANCY MANAGEMENT Contraception}

There is no evidence that oestrogen at doses contained in the combined contraceptive pill has an adverse effect on $\mathrm{MS}^{22} \mathrm{~A}$ single study did suggest a slightly lower rate of MS onset in women taking the pill, although this difference was not statistically significant. ${ }^{23}$ Oestrogen-containing oral contraceptives have been shown to suppress EAE in rats. ${ }^{24}$ Caution is advised only in patients at increased risk of deep venous thrombosis due to immobility.

\section{Conception}

MS appears to have no physiological effect on fertility, ${ }^{25}$ although sexual dysfunction may impact conception. Studies have highlighted the high prevalence of symptoms of sexual dysfunction in both male (around 65\%) and female (around $40 \%$ ) patients with MS compared with around $10 \%$ of case controls. ${ }^{26}$ These symptoms are highly under-reported during routine care. ${ }^{27}$

\section{Inheritance}

Susceptibility to multiple sclerosis is predicated upon a complex interaction between many individual genes and the external environment. The risk to offspring is small but can cause concern. Counselling for families with multiple sclerosis has been extensively reviewed elsewhere. ${ }^{28}{ }^{29}$ Overall, parents can be reassured that the age-adjusted lifetime risk of an offspring developing multiple sclerosis is only around $4 \%$ if one parent has MS (the same rate as the frequency of birth defects in the population), although this is significantly greater than the background population risk of $0.2 \%$. The rate can, however, be as high as $30 \%$ if both parents are affected.

\section{Pregnancy outcome}

Using the Norwegian Medical Birth Registry between 1967 and 2002, the outcomes of 649 births to women with MS were compared with births ( $\sim 2$ million) in the remaining population. Higher rates of operative deliveries were seen in the MS group (OR 1.54); these included an increase in elective caesarean section together with an increase in unplanned instrumental deliveries. Slow progress in the second stage of labour was seen twice as frequently in MS patients, and there were increased rates of labour induction. The proportion of neonates small for gestational age was also increased (OR 1.45). However, there was no difference in Apgar scores, birth defects or perinatal mortality. ${ }^{30}$ 
There is no evidence showing that patients with MS are at higher risk of developing stress-related urinary incontinence postpartum despite the potential for a longer second stage and slightly higher rate of instrumentation. Mode of delivery did not appear to influence the frequency of urinary disorders in a study of 273 MS patients having at least one pregnancy. ${ }^{31}$

\section{MS THERAPIES AND PREGNANCY}

Information on the risks of prednisolone and methylprednisolone in pregnancy is limited. The FDA places these drugs in group C (no adequate human or animal studies), though there have been concerns in the past that oral steroids in pregnancy might increase the risk of oral clefts. ${ }^{32}$ Greater experience now suggests, however, that this risk, if it exists, is small, and they are generally considered relatively safe. Theoretical concerns about fetal/neonatal adrenal suppression have not been borne out. Any fetal risks are likely to be small, primarily because this form of steroid is largely metabolised by the placenta so that very little reaches the fetus.

Current guidelines suggest stopping DMTs 3 months prior to conception for planned pregnancies. When conception on DMTs does occur, management is discussed with patients on a case-by-case basis, though the general recommendation is that they should consider stopping therapy due to a lack of evidence of safety. Animal data using 40 times the equivalent dose do not result in teratogenicity but do have an abortive effect. ${ }^{33}$ Exposure data are few for disease modifying agents in patients with MS. A review of 41 pregnancies from 3361 MS patients across eight interferon- $\beta$ (IFN) trials with in utero exposure suggested a non-significant increase in the rate of spontaneous abortion compared with population estimates but no overall increase in pregnancy loss or increased rate of anomaly. ${ }^{34} \mathrm{~A}$ second report in the same journal of 23 IFN exposed pregnancies found a higher rate of pregnancy loss and lower birth weight (200 g, similar to that seen in smokers). ${ }^{35}$ The degree to which the large molecule IFN crosses the placental barrier is not known, although it was not detectable in the fetal blood from two women taking IFN. ${ }^{36}$ In the end, it might be argued that the advice to stop IFN use in pregnancy is strengthened by the knowledge that the risk of relapse on stopping DMTs may well be mitigated by the natural reduction in relapse rate associated with pregnancy itself.

Other drugs occasionally used in MS may also need to be considered. Azathioprine has not been associated with an increased risk of congenital malformations and is considered relatively safe in pregnancy. Methotrexate should be avoided because of its known abortifacient effects and risk of malformations. The need for other symptomatic medication such as antispasticity or neuropathic pain medication should be reviewed with the patient on an individual basis prior to pregnancy.

\section{MANAGEMENT OF PREGNANCY IN MS PATIENTS}

Questions regarding management during pregnancy can arise for the neurologist, particularly if the patient with MS has significant neurological disability. Overall, patients can be reassured that the majority will have no particular difficulties during their pregnancy, labour and delivery. Occasional difficulties similar to those seen in patients with spinal cord injury can occur in MS patients, particularly those with spinal variants of $\mathrm{MS}$ or in those undertaking pregnancy with very advanced MS.
Some MS patients with existing mobility difficulties report further reduction in mobility and increased spasticity as the pregnancy progresses due to increasing weight and changes in the centre of gravity. They should also be warned against falls and may need increased physical therapy.

Pregnancy is a prothrombotic state, and immobile pregnant women are at significant risk of thromboembolism. Thromboprophylaxis with compression stockings, low-dose aspirin or heparin may need to be considered.

Urinary tract infections (UTIs) with ureteric reflux and pyelonephritis are more common during pregnancy, particularly with a pre-existing neurogenic bladder and may require an increase in the frequency of intermittent catheterisation. Frequent or chronic urinary tract infections can also increase the risk of preterm labour (PTL) (ie, labour before 37 weeks of gestation), and some women with recurrent UTIs may need long-term low-dose prophylactic antibiotics for the duration of the pregnancy. Women with MS are not otherwise at a higher risk of preterm labour but may be less able to detect the symptoms of PTL if there is significant spinal involvement above T6. These patients should be aware of this possibility and in severe cases have instruction on uterine palpation techniques or consider home monitoring with a portable uterine activity sensor.

There are case reports of autonomic dysreflexia (ADR) in patients with advanced MS. ${ }^{37}$ The risk is highest in patients with significant spinal cord pathology above T6, the level of splanchnic outflow, but can occur at lower levels. Loss of descending inhibitory spinal cord input and exacerbated afferent stimuli leads to sympathetic overactivity with vasoconstriction and hypertension below the level of spinal pathology and compensatory symptoms of parasympathetic overactivity (profuse diaphoresis, flushing and nasal congestion) above. Hypertension can be severe enough to precipitate seizures if untreated. Management is primarily through the awareness of precipitants (cold stirrups, vaginal examinations, catheter blockage) and early recognition (20-40 mm Hg systolic rise above baseline, headache and flushing). Rarely, pharmacological intervention with oral nifedipine may be required together with expedited delivery. Early use of epidural anaesthesia may be advocated in those at higher risk of $\mathrm{ADR}^{38}$ particularly if induction of labour is being considered.

There are usually no other particular issues during labour in patients with MS. The uterus is under neurohormonal control, so contractions are not adversely affected by spinal pathology in MS. The first stage of labour usually progresses as normal. Once the fetus descends into the pelvis, a minority of patients may require assistance with forceps or ventouse if pushing is impaired. While an increase in instrumental delivery rates was seen in the PRIMS study (3.4\% to 7\%), this was small. There is no evidence that epidural anaesthesia is contraindicated in patients with MS.

\section{POSTPARTUM}

Breastfeeding is usually unaffected in patients with multiple sclerosis. Occasionally, reduced milk production may occur after 6 weeks if there is reduced nipple stimulation secondary to sensory impairment. It is not known if $\beta$-interferon or copolymer-1 passes into breast milk, but it is advised that these are not used during breast-feeding. This consideration must be weighed against the desire to restart DMTs as early as possible in the postpartum period to minimise postpartum relapse risk.

Short courses of methylprednisolone are not contraindicated during breast-feeding. Only small concentrations are excreted in 
breast milk, and it has a short half-life. Breast-feeding does not impact on the risk of relapse within the postpartum period. ${ }^{39}$

\section{SUMMARY}

Women with multiple sclerosis should be reassured that pregnancy does not appear to be harmful overall and may even be beneficial. The perceived adverse impact on disease activity of the recommendation to stop disease modifying therapy prior to pregnancy may potentially be offset by the immunosuppressive effect of pregnancy itself. Treatment beyond DMT to reduce the heightened risk of relapse postpartum is not currently recommended but may deserve further consideration. The outcome of pregnancy for the majority of patients with MS is not significantly different from that of the general population, though some precautions may be required in patients with advanced or spinal forms of MS. Further understanding of the mechanism behind reduced MS disease activity during pregnancy may have implications for treating autoimmune diseases in general.

Competing interests: None.

\section{REFERENCES}

1. Mohr DC, Hart SL, Julian L, et al. Association between stressful life events and exacerbation in multiple sclerosis: a meta-analysis. BMJ 2004;328:731.

2. Tilman AJB. The effect of pregnancy on multiple sclerosis and its management. Res Publ Assoc Res Nerv Ment Dis 1950:28:548-82.

3. Wucherpfennig KW, Strominger JL. Selective binding of self peptides to diseaseassociated major histocompatibility complex (MHC) molecules: a mechanism for MHC-linked susceptibility to human autoimmune disease. J Exp Med 1995;181:1597-601.

4. Fujinami RS, von Herrath MG, Christen U, et al. Molecular mimicry, bystander activation, or viral persistence: infections and autoimmune disease. Clin Microbiol Rev 2006;19:80-94.

5. Crane IJ, Forrester JV. Th1 and Th2 lymphocytes in autoimmune disease. Crit Rev Immunol 2005;25:75-102.

6. Kim S, Liva SM, Dalal MA, et al. Estriol ameliorates autoimmune demyelinating disease: implications for multiple sclerosis. Neurology 1999:52:1220-8.

7. Sicotte NL, Liva R, Kluth $P$, et al. Treatment of multiple sclerosis with the pregnancy hormone estriol. Ann Neurol 2002;52:421-8.

8. Drew PD, Chavis JA. Female sex steroids: effects upon microglial cell activation. J Neuroimmunol 2000;111:77-85.

9. Wegman TG, Lin H, Guilbert $\mathrm{L}$, et al. Bidirectional cytokine interactions in the maternal-fetal reltionship: is successful pregnancy a TH-2 phenomenon. Immunol Today 1993;14:353-6.

10. Abramsky 0, Brenner T, Mizrachi R, et al. Alpha-fetoprotein suppresses EAE. J Neuro-immunol 1982;2:1-7.

11. Huizinga TW, van der Linden MW, Deneys-Laporte V, et al. Interleukin-10 as an explanation for pregnancy induced flare in systemic lupus erythematosus and remission in rheumatoid arthritis. Rheumatology 1999;38:496-8.

12. Confavreux C, Hutchinson M, Hours MM, et al. Rate of pregnancy-related relapse in multiple sclerosis. N Engl J Med 1998;339:285-91.
13. VanAmerongen BM, Dijkstra CD, Lips $P$, et al. Multiple sclerosis and vitamin D: an update. Eur J Clin Nutr 2004;58:1095-109.

14. Wingerchuk DM, Lesaux J, Gice G, et al. A pilot study of oral calcitriol $(1,25$ dihydroxyvitamin $D_{3}$ ) for relapsing remitting multiple sclerosis. JNNP 2005:76:1294-6.

15. McAlpine D, Compston N. Some aspects of the natural history of disseminated sclerosis. $0 \mathrm{~J}$ Med 1952;21:135-67.

16. Thompson DS, Nelson LM, Burns A, et al. The effects of pregnancy in multiple sclerosis: a retrospective study. Neurology 1986;36:1097-9.

17. van Walderveen MA, Tas MW, Barkhof F, et al. Magnetic resonance evaluation of disease activity during pregnancy in multiple sclerosis. Neurology 1994;44:327-9.

18. de Seze J, Chapelotte M, Delalande $S$, et al. Intravenous corticosteroids in the postpartum period for reduction of acute exacerbations in multiple sclerosis. Mult Scler 2004; 10:596-7.

19. Haas J. High dose IVIG in the post partum period for prevention of exacerbations in MS [discussion 33S]. Mult Scler 2000;6(2 Suppl):18-20S.

20. Runmarker B, Anderson 0. Pregnancy is associated with a lower risk of onset and a better prognosis in multiple sclerosis. Brain 1995;118:253-61.

21. Weinshenker BG, Hader W, Carriere W, et al. The influence of pregnancy on disability from multiple sclerosis: a population-based study in Middlesex County, Ontario. Neurology 1989:39:1438-40.

22. Hernan MA, Hohol MJ, Olek MJ, et al. Oral contraceptives and the incidence of multiple sclerosis. Neurology 2000;55:848-54.

23. Villard-Mackintosh L, Vessy MP. Oral contraceptives and reproductive factors in multiple sclerosis incidence. Contraception 1993;47:161-8.

24. Aronson BG, Richamn DP. Effect of oral contraceptives on experimental demyelinating disease. Arch Neurol 1969:21:103-8.

25. Damek DM, Shuster EA. Pregnancy and multiple sclerosis: review. Mayo Clin Proc 1997:72:977-89.

26. Zorzon M, Zivadinov R, Bosco A, et al. Sexual dysfunction in multiple sclerosis: a case control study. I. Frequency and comparison of groups. Mult Scler 1999;5:418-27.

27. O'Sullivan SS, Hardiman 0. Detection rates of sexual dysfunction amongst patients with multiple sclerosis in an outpatient setting _ can this be improved? Ir Med J 2006;100:304-6.

28. Sadovnick AD, Dircks A, Ebers GC. Genetic counselling in multiple sclerosis: risks to sibs and children of affected individuals. Clin Genet 1999;56:118-22.

29. Dwosh E, Guimond C, Sadovnick AD. Reproductive counselling for MS: a rationale Int MS J 2003:10:52-9.

30. Dahl J, Myhr KM, Daltveit AK, et al. Pregnancy, delivery, and birth outcome in women with multiple sclerosis. Neurology 2005;65:1961-3.

31. Durufle A, Nicolas B, Petrilli $S$, et al. Effects of pregnancy and childbirth on the incidence of urinary disorders in multiple sclerosis. Clin Exp Obstet Gynecol 2006;33:215-18.

32. Carmichael SL, Shaw GM. Maternal corticosteroid use and risk of selected congenital anomalies. Am J Med Genet 1999;86:242-4.

33. Avonex. Product monograph. Mississauga: Biogen, 1998

34. Sandberg-Wollheim M, Frank D, Goodwin TM, et al. Pregnancy outcomes during treatment with interferon beta-1a in patients with multiple sclerosis. Neurology 2005; 65:802-6.

35. Boskovic R, Wide R, Wolpin j, et al. The reproductive effects of beta interferon therapy in pregnancy. A longitudinal cohort. Neurology 2005;65:807-11.

36. Pons J-C, Lebon P. Frydman R, et al. Pharmacokinetics of interferon-alpha in pregnant women and fetoplacental passage. Fetal Diagn Ther 1995;10:7-10.

37. Bateman AM, Goldish GD. Autonomic dysreflexia in multiple sclerosis. J Spinal Cord Med 2002:25:40-2.

38. Kuczkowski KM. Labour analgesia for the parturient with spinal cord injury: what does an obstetrician need to know? Arch Gynecol Obstet 2006;274:108-12.

39. Vukusic S, Hutchinson M, Hours M, et al. The Pregnancy in Multiple Sclerosis Group; Pregnancy in Multiple Sclerosis Group. Pregnancy and multiple sclerosis (the PRIMS study): clinical predictors of post-partum relapse. Brain 2004;127:1353-60. 